\title{
The Accuracy of Point-of-Care Ultrasound Performed by Emergency Physicians in Detecting Hydronephrosis in Patients with Renal Colic
}

Abdulaziz Al-Balushi, ${ }_{1}^{1}$ Amal Al-Shibli, ${ }^{2}$ Abdullah Al-Reesi, ${ }^{2}$ Qazi Zia Ullah, ${ }^{2}$ Waleed Al-Shukaili, ${ }^{2}$ Saleh Baawain, Hamoud Al-Dhuhli, ${ }^{3}$ Mohammed Al-Shamsi, ${ }^{4}$ Ahmed Al-Hubaishi, ${ }^{5}{ }^{*} \mathrm{Al}$ Yaqdhan H. Al-Atbi ${ }^{6}$

\begin{abstract}
Objectives: This study aimed to determine the accuracy of renal point-of-care ultrasound (POCUS) performed by emergency physicians in detecting hydronephrosis in patients with renal colic. Methods: This prospective cross-sectional diagnostic study was conducted at the Emergency Department (ED) of Sultan Qaboos University Hospital from February 2017 to February 2018. All adult patients with suspected renal colic and who could give informed consent were included. The emergency physicians performed POCUS on both kidneys and graded hydronephrosis as none, mild, moderate or severe. The POCUS grade was compared to the non-contrast computed tomography (NCCT) grade. Results: A total of 303 participants were enrolled in the analysis. The majority of the study population comprised adult males (247 [81.5\%]), and the mean age was 39 years. Among the samples, $71.3 \%$ of the patients exhibited a degree of hydronephrosis based on CT findings. Ultrasounds performed by emergency physicians had a sensitivity of $75.8 \%$ (95\% CI: 69.5-81.4), specificity of 55.2\% (95\% CI: 44.1-65.8), a positive likelihood ratio of 1.69 (95\% CI: 1.32-2.16) and a negative likelihood ratio of 0.43 (95\% CI: $0.32-0.59$ ) for hydronephrosis using CT as the criterion standard. Conclusion: When evaluating patients with suspected renal colic, a bedside renal POCUS performed by emergency physicians has a moderate sensitivity to detect hydronephrosis and grade its severity. Therefore, it should be utilised in the ED to screen patients for hydronephrosis; however, more training is required to improve the test's accuracy.
\end{abstract}

Keywords: Hematuria; Hydronephrosis; Nephrolithiasis; Point-of-Care Testing; Renal Colic; Ultrasonography; Oman.

\section{ADVANCES IN KNOWLEDGE}

Renal point-of-care ultrasound (POCUS) performed by emergency physicians demonstrates moderate sensitivity in detecting hydronephrosis in patients who present to the emergency department with renal colic.

There is no correlation between the severity of hydronephrosis and ureteric jet.

\section{Application to Patient Care}

Renal POCUS is a quick, non-invasive, easily available and safe bedside diagnostic tool for renal stones.

Renal POCUS allows emergency physicians to improve their diagnostic accuracy and disposition and provide better overall patient care. Rapid screening for hydronephrosis in the emergency department using renal POCUS might exclude obstruction, help focus assessment on other potential diagnoses and allow physicians to select patients for further analyses.

$\mathrm{U}$ RINARY TRACT STONES ARE COMMON AMONG the general population. Around $12 \%$ of the population will have urinary tract stones during their lifetime. ${ }^{1}$ In the USA, a survey revealed an increase in the prevalence of renal stones among the population between 1980 and 2014. ${ }^{2}$ Additionally, acute renal colic is one of the common manifestations encountered in the Emergency Department (ED). In this regard, reports have shown that around 1-2 million ED visits per year in the USA are due to renal colic. ${ }^{2-4}$ It is described as a sudden onset of severe flank pain that radiates to the groin. This pain is usually caused by a ureteric stone. ${ }^{5}$

Hydronephrosis is defined as the dilatation of the renal pelvis and calyces. It is an indirect sign of distal obstruction in the urinary tract system. ${ }^{6}$ Based on severity, hydronephrosis is categorised into four grades: none, mild, moderate and severe. ${ }^{7}$ Mild hydronephrosis is defined as the dilation of the renal pelvis only. Moderate hydronephrosis is defined as the dilatation of the pelvi-calyceal system without renal cortical thinning. Severe hydronephrosis is defined as the dilatation of the pelvi-calyceal system associated with cortical thinning. ${ }^{7-9}$

Non-contrast computed tomography (NCCT) is considered the gold standard for the diagnosis of urolithiasis and hydronephrosis in ED patients. ${ }^{10-12}$ However, it is costly and time consuming and exposes patients to significant doses of radiation. ${ }^{13}$ In contrast, point-of-care ultrasound (POCUS) is a non-invasive,

${ }^{1}$ Department of Emergency Medicine, Sohar Hospital, Sohar, Oman; Departments of ${ }^{2}$ Accident E Emergency and ${ }^{3}$ Radiology, Sultan Qaboos University Hospital, Muscat, Oman; ${ }^{4}$ Department of Emergency Medicine, Armed Forces Hospital, Muscat, Oman; ${ }^{5}$ Department of Emergency Medicine, Royal Hospital, Muscat, Oman; Department of Emergency Medicine, Royal Oman Police Hospital, Muscat, Oman

*Corresponding Author's e-mail: a_alatbi@hotmail.com 
easily available, quick and safe bedside procedure that does not pose any radiation risk. ${ }^{14}$ Furthermore, it allows emergency physicians (EP) to improve their diagnostic accuracy and disposition and provide better overall patient care. ${ }^{14}$ Rapid ED screening for hydronephrosis and absence of ureteric jet might exclude obstruction and help focus assessment on other potential diagnoses and might allow the selection of patients for further analyses.

The literature supporting the use of ultrasound by EPs to detect hydronephrosis has been growing. Studies have demonstrated promising results regarding the sensitivity and specificity of renal POCUS performed by EPs in detecting hydronephrosis. The sensitivity ranges from $72 \%$ to $80 \%$ and the specificity ranges from $73 \%$ to $83 \%{ }^{15-17}$

When the urine reaches the terminal portion of the ureter, it gets ejected forcefully into the urinary bladder through the vesicoureteric junction (VUJ). The visualisation of the urine efflux using colour Doppler ultrasound from the distal end of each ureter into the urinary bladder is called the ureteric jet. ${ }^{18}$

Studies have demonstrated that there is a direct relationship between ureteric jet and the presence of obstructing ureteric stones. ${ }^{19-21}$ One such study showed that the ureteric jet in an obstructed ureter has less frequency, shorter duration and lower peak velocity when compared with the contralateral normal side. ${ }^{20}$ Nevertheless, the study determined that the presence of a ureteric jet did not rule out significant ureteric obstruction. ${ }^{21}$

Primarily, this study aimed to investigate the accuracy of renal POCUS performed by EPs in detecting hydronephrosis in patients with renal colic. Additionally, the authors hypothesised that the presence of high-grade hydronephrosis indicates an obstructive ureteric stone, which will result in the absence of a ureteric jet. Therefore, the study's secondary aim was to assess the correlation between ureteric jet and the severity of hydronephrosis.

\section{Methods}

A prospective cross-sectional diagnostic study was conducted at the ED of Sultan Qaboos University Hospital (SQUH) from February 2018 to February 2019. SQUH is a tertiary academic hospital with an annual ED visit of more than 60,000 patients.

The study included all adult patients who were suspected to have renal colic, were aged more than 18 years old, were not known to have a renal disease (chronic kidney disease, polycystic kidney disease, etc.), were not enrolled previously in the study, had renal NCCT done within 24 hours after bedside ultrasound and gave written consent to be included in the study. The study excluded pregnant patients, female patients aged less than 40 years old and patients who were not able/refused to give consent. The reason female patients aged less than 40 years old were excluded is that the ED protocol for them mandates doing departmental ultrasound as the first step rather than renal NCCT.

The sample size was calculated based on previous studies that reported the incidence of renal colic. Using a confidence interval of $95 \%$, the sample size was estimated to be 300 participants.

All scans were done by POCUS-certified EPs. An extra one-hour renal POCUS session was conducted for all providers prior to the start of the study to achieve agreement regarding the labelling and degree of hydronephrosis. The level of those physicians varied between emergency residents, specialists and consultants.

After obtaining consent, the EPs performed renal POCUS on both kidneys using a low-frequency (2-5 $\mathrm{MHz}$ ) curvilinear probe. The severity of hydronephrosis was then classified as none, mild, moderate or severe [Figure 1].

Then, the renal POCUS grade was compared to the NCCT grade, which was interpreted by a senior radiologist blinded to the renal POCUS results. The NCCT was performed less than 24 hours from the POCUS. The flow chart of the study was distributed to the triage nurses, emergency doctors and radiology technicians [Figure 2].

The ultrasound images and videos were saved and reviewed by an independent investigator who is an emergency medicine consultant and POCUS instructor. This review aimed to ensure that the operators were obtaining the optimum images of the kidneys. After the review, feedback was delivered to individual operators about their performance.

The data collection sheet included information on patient demographics, the level of the EPs (the operators), the site of the flank pain, the presence or absence of red blood cells (RBCs) in the urine dipstick, the degree of hydronephrosis based on POCUS findings and the degree of hydronephrosis based on the NCCT findings. The operators identified their findings regarding the degree of hydronephrosis, marked them on the data collection sheet and then dropped the sheet in a sealed box. An independent investigator collected the data sheets later and entered them into EpiData (The EpiData Association, Odense, Denmark). NCCT data about the presence and degree of hydronephrosis, as well as the presence, size and 



Figure 1: Grading hydronephrosis severity using point-of-care ultrasound as (A) mild, (B) moderate and (C) severe.

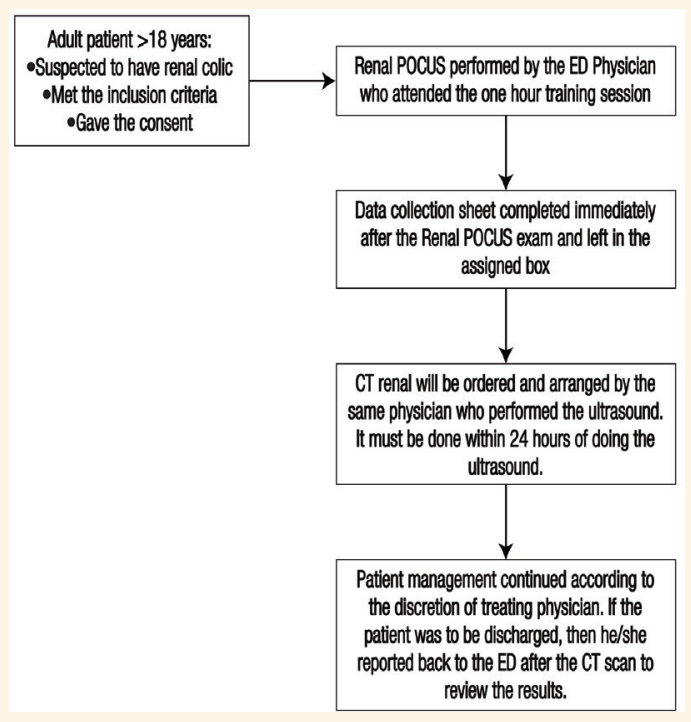

Figure 2: Flowchart of study procedure.

POCUS = point-of-care ultrasound; $C T=$ computed tomography; $E D=$ emergency department

location of ureteral stones, were abstracted from the dictated radiology report by an investigator blinded to the renal POCUS results. The data were analysed using the Statistical Package for the Social Sciences (SPSS), Version 22 (IBM Corporation, Armonk, New York, USA).

Ethical approval was obtained from the Medical Research Ethics Committee, College of Medicine and Health Sciences, at Sultan Qaboos University.

\section{Results}

A total of 303 participants were enrolled in the analysis. The majority of the study population comprised adult males (247 [81.5\%]) and the mean age was 39 years. Emergency medicine specialists performed around $67 \%$ of the renal POCUS, while residents and consultants did the rest. Hydronephrosis was present in 216 (71.3\%) NCCT scans and ureteral stones were present in 207 (68.3\%) NCCT reports. However, hydronephrosis without ureteral stones was noted in nine (3.0\%) NCCT scans [Table 1].
Table 1: Characteristics of the study participants $(\mathrm{N}=303)$

$\begin{array}{lc}\text { Characteristic } & \mathbf{n}(\%) \\ \text { Mean age in years } & 39 \\ \text { Number of male participants } & 247(81.5) \\ \text { Haematuria in urine dipstick } & 234(78.8) \\ \text { Hydronephrosis present on renal POCUS } & 205(67.5) \\ \text { Hydronephrosis present on NCCT } & 215(71) \\ \text { Hydronephrosis POCUS grade } & \\ \text { None } & 87(28.7) \\ \text { Mild } & 78(25.7) \\ \text { Moderate } & 138(45.5) \\ \text { Severe } & 0(0.0) \\ \text { Ureteral stone present on NCCT } & 207(68.3) \\ \text { Hydronephrosis without ureteral stone } \\ \text { on NCCT } & 9(3.0) \\ \text { Ureteric jet } & \\ \text { Present } & 135(44.6) \\ \text { Absent } & 168(55.5)\end{array}$

POCUS $=$ point-of-care ultrasound $;$ NCCT $=$ non-contrast computed tomography.

Table 2: Sensitivity, specificity and likelihood ratio for the presence of hydronephrosis; comparing bedside renal point-of-care ultrasound to non-contrast computed tomography

\begin{tabular}{|c|c|}
\hline \multicolumn{2}{|c|}{ Overall population } \\
\hline $\begin{array}{l}\text { Sensitivity: } 75.8 \% \\
\text { (95\% CI: } 69.5-81.4)\end{array}$ & $\begin{array}{l}\text { Positive Likelihood Ratio: } 1.69 \\
\text { ( } 95 \% \text { CI: } 1.32-2.16)\end{array}$ \\
\hline $\begin{array}{l}\text { Specificity: } 55.2 \% \\
\text { (95\% CI: } 44.1-65.8 \text { ) }\end{array}$ & $\begin{array}{l}\text { Negative Likelihood Ratio: } 0.43 \\
\text { (95\% CI: 0.32-0.59) }\end{array}$ \\
\hline \multicolumn{2}{|c|}{ Mild Hydronephrosis } \\
\hline $\begin{array}{l}\text { Sensitivity: } 58.33 \% \\
\text { (95\% CI: } 44.88 \%-70.93 \%)\end{array}$ & $\begin{array}{l}\text { Positive Likelihood Ratio: } 1.49 \\
(95 \% \text { CI: } 1.05-2.10)\end{array}$ \\
\hline $\begin{array}{l}\text { Specificity: } 60.76 \% \\
\text { (95\% CI: } 49.12 \%-71.56 \%)\end{array}$ & $\begin{array}{l}\text { Negative Likelihood Ratio: } 0.68 \\
\text { (95\% CI: 0.48-0.97) }\end{array}$ \\
\hline \multicolumn{2}{|c|}{ Moderate Hydronephrosis } \\
\hline $\begin{array}{l}\text { Sensitivity: } 64.93 \% \\
\text { (95\% CI: } 53.21 \%-75.46 \%)\end{array}$ & $\begin{array}{l}\text { Positive Likelihood Ratio: } 4.54 \\
\text { ( } 95 \% \text { CI: } 2.34-8.81 \text { ) }\end{array}$ \\
\hline $\begin{array}{l}\text { Specificity: } 85.71 \% \\
(95 \% \text { CI: } 73.78 \%-93.62 \%)\end{array}$ & $\begin{array}{l}\text { Negative Likelihood Ratio: } 0.41 \\
\text { ( } 95 \% \text { CI: } 0.30-0.65 \text { ) }\end{array}$ \\
\hline
\end{tabular}




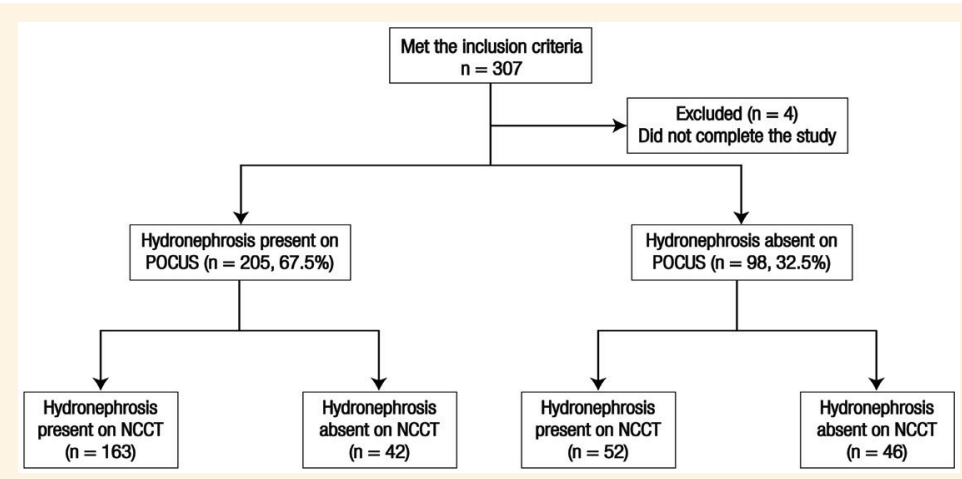

Figure 3: A diagram of test characteristics of any hydronephrosis on ultrasonography compared with any hydronephrosis on CT.

POCUS = point-of-care ultrasound; NCCT = non-contrast computed tomography.

Furthermore, $21 \%$ of the participants were found to have negative RBCs on urine dipstick despite the presence of renal stones in NCCT. The mean time between the renal POCUS and NCCT scan was four hours. Figure 3 displays the characteristics of any hydronephrosis on POCUS compared with any hydronephrosis on NCCT, using the Standards for Reporting of Diagnostic Accuracy (STARD) format. ${ }^{22}$

On the other hand, hydronephrosis detected on renal POCUS exhibited $75.8 \%$ sensitivity and 55.2\% specificity, with a positive likelihood ratio of 1.69 (95\% CI: 1.32-2.16) and a negative likelihood ratio of 0.43 (95\% CI: 0.32-0.59), using hydronephrosis on NCCT as the criterion standard. Patients with mild hydronephrosis had a sensitivity of $58.33 \%$ (95\% CI 44.88-70.93\%), whereas patients with moderate hydronephrosis had a sensitivity of $64.93 \%$ (95\% CI 53.21-75.46\%) [Table 2].

Although five cases were labelled as severe hydronephrosis on POCUS, none were found on NCCT. Based on the NCCT findings, 87 participants (28.7\%) had no hydronephrosis, while 78 (25.7\%) and 138 (45.5\%) patients had mild and moderate hydronephrosis, respectively [Table 2].

The ureteric jet was absent in $74.2 \%$ of patients who had hydronephrosis and ureteric stones on NCCT. The absence of ureteric jet was observed in $51.1 \%$ of patients with mild hydronephrosis and $64.6 \%$ of patients with moderate hydronephrosis.

\section{Discussion}

The findings of this study demonstrated that around two-thirds of patients who presented to the ED with the signs and symptoms of renal colic had hydronephrosis on NCCT. This was slightly higher than a prospective study of 670 patients which reported that the rate of hydronephrosis among patients with acute renal colic was $47.5 \%{ }^{16}$
In the current study, the sensitivity of renal POCUS performed by EPs was 75.8\% (95\% CI: 69.5-81.4), which aligned with the findings of previous studies. ${ }^{15,23}$ In this regard, Herbst et al. studied 670 patients with renal colic and found that the sensitivity to detect hydronephrosis was $72.6 \%$ when performed by EPs. ${ }^{16}$ Notably, in that study, the sensitivity of renal POCUS improved as the degree of hydronephrosis increased.

Furthermore, the current study demonstrated that renal POCUS has a specificity of $55.2 \%$ (95\% CI: 44.1-65.8) in detecting hydronephrosis when performed by EPs. This result varied from previous studies that reported the specificity of renal POCUS to be ranging from $60 \%$ to $83 \%$. $^{7,15,16}$ This variation can be attributed to the experience level of the ultrasound operator and the ultrasonic findings that mimic hydronephrosis. For instance, 32 (10\%) participants in the current study were found to have cases such as extra-renal pelvis and cysts that mimic hydronephrosis and were thus interpreted as hydronephrosis.

To the best of the authors' knowledge, there are no previously published studies that examine the relationship between the degree of hydronephrosis and the ureteric jet. In this regard, the study found that no direct relationship is present between the grade of hydronephrosis and ureteric jet. Among patients who were identified to have moderate hydronephrosis in the NCCT scan (100 patients), ureteric jet was present in $58 \%$ and absent in $42 \%$.

The absence of microscopic haematuria does not eliminate renal colic as a potential diagnosis. In this sense, haematuria was not present in $21 \%$ of participants who had renal stones on CT scans. This finding aligns with those of previously published studies. ${ }^{24,25}$

This study has a few limitations. First, although the mean time between performing the POCUS and the renal NCCT was four hours, there were several cases 
where the NCCT scan was done around 10-12 hours after having renal POCUS done. Hence, maintaining a constant time frame between performing the renal POCUS and the NCCT will eliminate the possibility of change in the degree of hydronephrosis from mild hydronephrosis to no hydronephrosis and vice versa. Second, all the operators underwent a one-hour ultrasound training session only, including a lecture and hands-on practice. Increasing the duration of the training session might improve the sensitivity and specificity. Third, the study was conducted at SQUH, which is one of the major academic tertiary hospitals in the country and POCUS was introduced earlier to the ED than in other hospitals. Therefore, it is difficult to generalise the study outcomes to all other hospitals.

\section{Conclusion}

When evaluating patients with suspected renal colic, a bedside renal POCUS performed by EPs has a moderate sensitivity to detect hydronephrosis and grade its severity. Therefore, the utilisation of POCUS in the ED to screen patients for hydronephrosis is recommended. Furthermore, there is no correlation between the severity of hydronephrosis and ureteric jet. More training and interventional studies are required to improve the accuracy of the test at SQUH, as it might serve as a helpful screening tool in the ED.

\section{AUTHORS' CONTRIBUTIONS}

$\mathrm{AB}, \mathrm{AS}, \mathrm{AR}, \mathrm{HD}, \mathrm{MS}$ and $\mathrm{AH}$ conceptualised and designed the study. AS and MS provided a training session to the investigators of the study. QZ and WS collected the data. WS performed the statistical analysis. $\mathrm{AB}, \mathrm{AR}, \mathrm{SB}$ and $\mathrm{AH}$ analysed and interpreted the data. $A B$ and AS drafted the manuscript. AR, MS and AA critically revised the manuscript. All authors approved the final version of the manuscript.

\section{CONFLICT OF INTEREST}

The authors declare no conflicts of interest.

\section{FUNDING}

No funding was received for this study.

\section{References}

1. Teichman JMH. Clinical practice. Acute renal colic from ureteral calculus. N Engl J Med 2004; 350:684-93. https://doi.org/10.10 56/NEJMcp030813.

2. Feit L, John D, Delgado Torres N, Sinert R. Flank pain and hematuria is not always a kidney stone. Am J Emerg Med 2021; 40:225.e1-2. https://doi.org/10.1016/j.ajem.2020.07.046.
3. Schoenfeld EM, Shieh M-S, Pekow PS, Scales CD, Munger JM, Lindenauer PK. Association of patient and visit characteristics with rate and timing of urologic procedures for patients discharged from the emergency department with renal colic. JAMA Netw Open 2019; 2: e1916454. https://doi.org/10.1001/ jamanetworkopen.2019.16454.

4. Daniels B, Gross CP, Molinaro A, Singh D, Luty S, Jessey R, et al. STONE PLUS: Evaluation of emergency department patients with suspected renal colic, using a clinical prediction tool combined with point-of-care limited ultrasonography. Ann Emerg Med 2016; 67:439-48. https://doi.org/10.1016/j. annemergmed.2015.10.020.

5. ClinicalKey. Nephrolithiasis. From: https://www.clinicalkey. com/\#!/content/book/3-s2.0-B9781455753598000080 Accessed: Jul 2021.

6. Thotakura R, Anjum F. Hydronephrosis and hydroureter In: StatPearls [Internet]. Treasure Island, USA: StatPearls Publishing, 2020. From: http://www.ncbi.nlm.nih.gov/books/ NBK563217/ Accessed: Jul 2021.

7. Pathan SA, Mitra B, Mirza S, Momin U, Ahmed Z, Andraous LG, et al. Emergency physician interpretation of point-of-care ultrasound for identifying and grading of hydronephrosis in renal colic compared with consensus interpretation by emergency radiologists. Acad Emerg Med 2018; 25:1129-37. https://doi. org/10.1111/acem.13432.

8. Onen A. Grading of hydronephrosis: An ongoing challenge. Front Pediatr 2020; 8:458. https://doi.org/10.3389/fped.2020.0 0458 .

9. Nguyen HT, Benson CB, Bromley B, Campbell JB, Chow J, Coleman B, et al. Multidisciplinary consensus on the classification of prenatal and postnatal urinary tract dilation (UTD classification system). J Pediatr Urol 2014; 10:982-98. https:// doi.org/10.1016/j.jpurol.2014.10.001.

10. Fulgham PF, Assimos DG, Pearle MS, Preminger GM. Clinical effectiveness protocols for imaging in the management of ureteral calculous disease: AUA technology assessment. J Urol 2013; 189:1203-13. https://doi.org/10.1016/j.juro.2012.10.031.

11. Brisbane W, Bailey MR, Sorensen MD. An overview of kidney stone imaging techniques. Nat Rev Urol 2016; 13:654-62. https://doi.org/10.1038/nrurol.2016.154.

12. Coursey CA, Casalino DD, Remer EM, Arellano RS, Bishoff JT, Dighe $\mathrm{M}$, et al. ACR appropriateness criteria ${ }^{\circledR}$ acute onset flank pain--suspicion of stone disease. Ultrasound Q 2012; 28:227-33. https://doi.org/10.1097/RUQ.0b013e3182625974.

13. Nicolau C, Claudon M, Derchi LE, Adam EJ, Nielsen MB, Mostbeck G, et al. Imaging patients with renal colic-consider ultrasound first. Insights Imaging 2015; 6:441-7. https://doi. org/10.1007/s13244-015-0396-y.

14. Kim SG, Jo IJ, Kim T, Hwang SY, Park JH, Shin TG, et al. Usefulness of protocolized point-of-care ultrasonography for patients with acute renal colic who visited emergency department: A randomized controlled study. Medicina (Kaunas) 2019; 55:717-28. https://doi.org/10.3390/medicina55110717.

15. Watkins S, Bowra J, Sharma P, Holdgate A, Giles A, Campbell L. Validation of emergency physician ultrasound in diagnosing hydronephrosis in ureteric colic. Emerg Med Australas 2007; 19:188-95. https://doi.org/10.1111/j.1742-6723.2007.00925.x.

16. Herbst MK, Rosenberg G, Daniels B, Gross CP, Singh D, Molinaro AM, et al. Effect of provider experience on clinicianperformed ultrasonography for hydronephrosis in patients with suspected renal colic. Ann Emerg Med 2014; 64:269-76. https://doi.org/10.1016/j.annemergmed.2014.01.012.

17. Moak JH, Lyons MS, Lindsell CJ. Bedside renal ultrasound in the evaluation of suspected ureterolithiasis. Am J Emerg Med 2012; 30:218-21. https://doi.org/10.1016/j.ajem.2010.11.024. 
18. Leung VY, Chu WC, Yeung C, Metreweli C. Doppler waveforms of the ureteric jet: An overview and implications for the presence of a functional sphincter at the vesicoureteric junction. Pediatr Radiol 2007; 37:417-25. https://doi.org/10.1007/s00247-007-0433-1.

19. de Bessa J, Dénes FT, Chammas MC, Cerri L, Monteiro EDS, Buchpiguel CA, et al. Diagnostic accuracy of color Doppler sonographic study of the ureteric jets in evaluation of hydronephrosis. J Pediatr Urol 2008; 4:113-7. https://doi.org/10.10 16/j.jpurol.2007.10.013

20. Jandaghi AB, Falahatkar S, Alizadeh A, Kanafi AR, Pourghorban R, Shekarchi B, et al. Assessment of ureterovesical jet dynamics in obstructed ureter by urinary stone with color Doppler and duplex Doppler examinations. Urolithiasis 2013; 41:159-63. https://doi.org/10.1007/s00240-012-0542-7.

21. Kuzmić AC, Brkljacić B, Rados M, Galesić K. Doppler visualization of ureteric jets in unilateral hydronephrosis in children and adolescents. Eur J Radiol 2001; 39:209-14. https://doi. org/10.1016/s0720-048x(01)00329-1.
22. Bossuyt PM, Reitsma JB, Bruns DE, Gatsonis CA, Glasziou PP, Irwig LM, et al. Towards complete and accurate reporting of studies of diagnostic accuracy: The STARD Initiative. Clin Radiol 2003; 58:575-80. https://doi.org/10.1016/s0009-9260(03)00258-7.

23. Riddell J, Case A, Wopat R, Beckham S, Lucas M, McClung CD, etal. Sensitivity of emergencybedside ultrasound to detecthydronephrosis in patients with computed tomography-proven stones. West J Emerg Med 2014; 15:96-100. https://doi.org/10.5811/ westjem.2013.9.15874.

24. Leslie SW, Sajjad H, Murphy PB. Renal Calculi. In: StatPearls [Internet]. Treasure Island, USA: StatPearls Publishing. From: http://www.ncbi.nlm.nih.gov/books/NBK442014/ Accessed: Jul 2021.

25. Mefford JM, Tungate RM, Amini L, Suh D, Anderson CL, Rudkin SE, et al. A comparison of urolithiasis in the presence and absence of microscopic hematuria in the emergency department. West J Emerg Med 2017; 18:775-9. https://doi. org/10.5811/westjem.2017.4.33018. 